\title{
KYSTE AMYGDALOÏDE OROPHARYNGE A PROPOS D'UN CAS
}

\author{
H. HAJRI, S. MANOUBI, N. KAFFEL, B. BOUZAIENE, K. RIAHI, \\ M. MARRAKCHI, D. NAJAH, R. LAHIANI, M. FERJAOUI \\ SERVICE ORL HÔPITAL CHARLES NICOLLE
}

\begin{abstract}
RESUME
Le kyste amygdaloïde est une tumeur kystique latéro-cervicale haute rare, issue de la 2ème fente branchiale. II représente $2 \%$ des tumeurs latéro-cervicales du cou, et $6,1 a ̀$ is, $2 \%$ des anomalies de la deuxième fente. La forme oropharyngée est très rare.

Nous rapportons une observation d'un kyste amygdaloïde oropharyngé, chez une femme de 56 ans. Aucune symptomatologie particulière n'a été notée.

L'examen clinique a révélé une tuméfaction de la loge amygdalienne droite, refoulant le pilier antérieur. La TDM a objectivé un processus expansif nécrosé de l'amygdale palatine droite comblant l'espace parapharyngé droit, en contact avec la base de la langue et le muscle ptérygoïdien médial homolatéral.

Une résection complète et sans rupture du kyste a été pratiquée par voie transorale, le geste a été complété par une amygdalectomie droite.

L'histologie a confirmé le diagnostic par la co-existence d'un revêtement épithélial et d'une infiltration de tissu lymphoïde. Le recul est de 6 mois sans récidive.
\end{abstract}

Mots clés : Appareil branchial, kyste, oropharynx.

\section{SUMMARY}

Second branchial cleft cysts are the most common type of branchial abnormalities and usually found high in the neck. Oropharyngeal presence of branchial cleft cyst is very rare. We report a case of oropharyngeal branchial cleft cyst in 56 years women, wich had any specific symptom. Radiologic examination (TDM) revealed an expansive mass of the palatin tonsil.

It was removed completely with right palatin tonsil.

Cyst had a squamous epithelium-lined wall with lymphoid aggregation in histopathologic study, which was chareacteristic finding of branchial cleft cyst. There was no evidence of recurrence for 6 months for follow-up. We review reported oropharyngeal or nasopharyngeal presentation of these cases in literature and embryologic explanation.

Key words: Oropharyngeal, branchial, cysts

\section{INTRODUCTION}

Les kystes amygdaloïdes comptent parmi les plus fréquentes des anomalies branchiales, ils représentent 6,1à $85,2 \%$ des anomalies de la deuxième fente [1]. Ils sont dus à la persistance du sinus cervical au cours de la différenciation de l'appareil branchial. Le siège habituel est le tiers moyen du bord antérieur du muscle sterno-cléido-mastoïdien mais ils peuvent se situer à n'importe quel point depuis le muscle constricteur moyen du pharynx à la région susclaviculaire.

Nous rapportons une observation rare de kyste amygdaloïde de siège pharyngien. Cette topographie rare s'explique par le développement embryologique de l'appareil branchial.

\section{OBSERVATION}

L'examen ORL d'une femme âgée de 56 ans qui a consulté pour otalgie a révélé la présence d'un bombement du pilier antérieur droit, refoulant l'amygdale palatine en dedans et en avant qui est d'aspect normal. La TDM a objectivé une formation hypodensbien limité comblant l'es- pace parapharyngé droit, en contact avec la base de la langue et le muscle ptérygoïdien médial homolatéral (Figure 1,2).

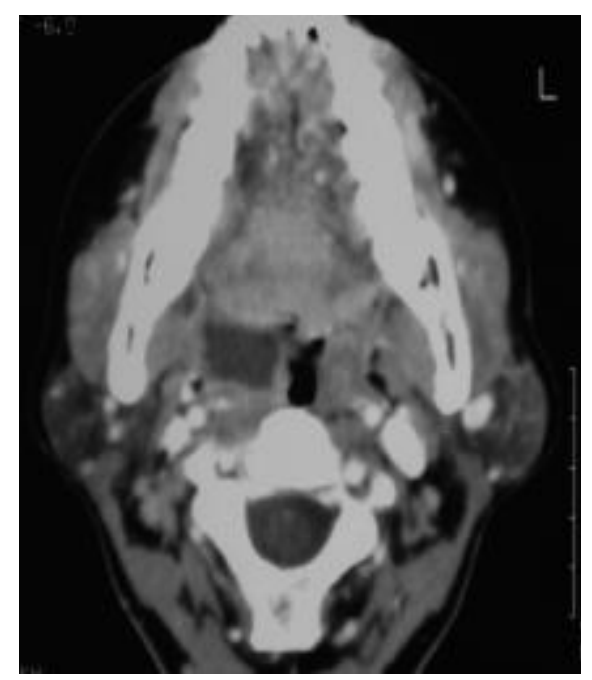

Fig. 1 : TDM coupe axiale :

formation kystique parapharyngée droite 


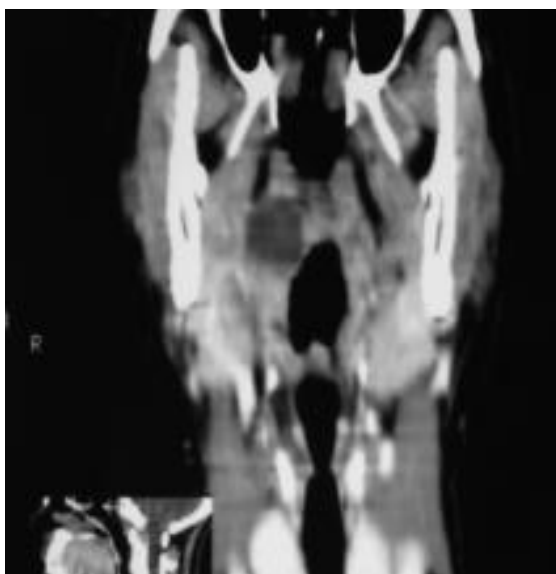

Fig. 2 : TDM coupe coronale :

formation hypodense parapharyngée droite

Devant le caractère kystique et la taille réduite de la tumeur nous avons opté pour la voie transorale. La résection a été complète, sans rupture d'un kyste bien encapsulé emportant en monobloc la tonsille palatine homolatérale. Nous n'avons pas retrouvé de fistule interne Figure 3.

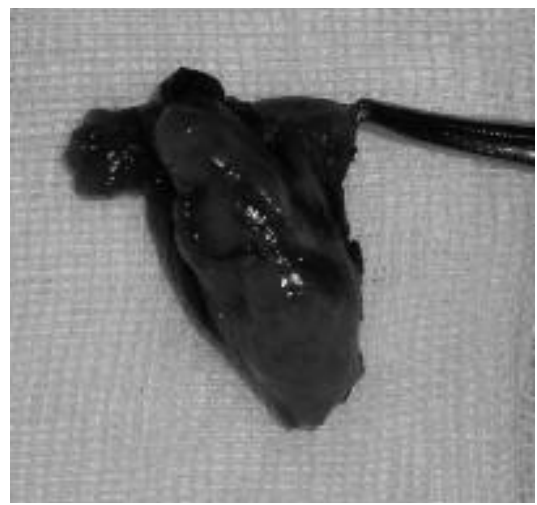

Fig. 3 : Pièce d'exérèse chirurgicale emportant le kyste et l'amygdale

L'étude histologique a confirmé le diagnostic, en montrant un revêtement épithélial pavimenteux stratifié avec une infiltration lymphoïde Figure 4.

Les suites opératoires étaient sans complications, le recul était de six mois sans récidive.

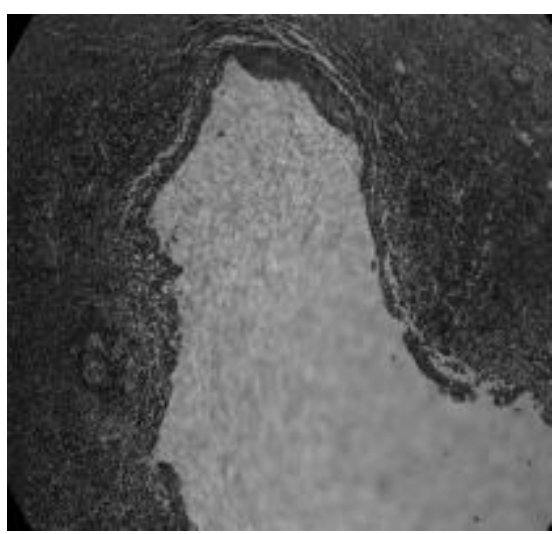

Fig. 4 : Revêtement épithélial malpighien : infiltration lymphoïde du chorion

\section{DISCUSSION}

Les tumeurs bénignes kystiques dysembryologiques se développent au niveau de la partie antéro-latérale du cou. Elles font partie des anomalies congénitales de la deuxième fente branchiale, la fréquence des kystes amygdaloïdes par rapport aux anomalies de la deuxième fente varie de 6,1 à $85,2 \%$ [1].

L'âge de découverte est plus avancé que pour les autres fistules congénitales, avec un pic de fréquence à la 2 ème et à la 3ème décade.

La topographie des kystes amygdaloïdes peut être expliquée par le siège du sinus cervical de His lequel normalement disparaît complètement, mais il peut persister occasionnellement sous forme d'un kyste cervical [2] Le kyste communique parfois avec la peau ou le pharynx, spontanément ou suite à une surinfection. La communication avec l'extérieur se fait par un canal étroit que l'on appelle fistule cervicale externe, dont l'orifice externe se situe souvent à la réunion du tiers moyen et tiers inférieur du bord antérieur du muscle sterno-cleido-mastoidien. Alors que la communication avec le pharynx, plus rare que la précédente, se fait par une fistule interne qui s'ouvre dans un dérivé embryonnaire de la deuxième poche branchiale, l'amygdale [3].

La clinique dépend de la localisation et de la taille du kyste.

Le kyste amygdaloïde est tapissé par un épithélium de différents types, le plus malpighien [4,5].

Il peut s'agir aussi d'un épithélium de type cylindrique cilié d'origine éctodermique. Certains auteurs pensent que la présence de kératine, la présence de tissu lymphoïde sont des critères obligatoires au diagnostic du kyste amygdaloïde [6].

La localisation pharyngienne est très rare. En 1993, Thaler a rapporté une observation rare de kyste amygdaloïde oropharyngé sans fistule [7]

Cette localisation est expliquée par l'apposition ectoblaste-entoblaste de la membrane obturatrice, en l'absence de l'arc mésoblastique sans qu'il y ait une communication via une fistule avec l'oropharynx [2], tel était le cas de notre patiente.

Chez l'adulte cette forme clinique est souvent confondue avec un abcès parapharyngé, le recours à l'imagerie (TDM/IRM) est indispensable pour étayer le diagnostic, mais la confirmation reste toujours chirurgicale et anatomopathologique. La TDM ou I'IRM sont particulièrement indiquées pour différencier la lésion des autres tumeurs parapharyngées :

Un hémangiome, lymphangiome ou kyste dermoide, adénopathie métastatique dont la distinction avec un kyste amygdaloïde dégénéré ou une métastase inta kystique est très difficile.

La TDM pratiquée chez notre patiente évoquait plutôt une tumeur parapharyngée, le diagnostic a été redressé par la chirurgie et l'histologie. 


\section{CONCLUSION}

Le kyste de la deuxième fente branchiale est une tumeur bénigne latéro-cervicale dysembryoplasique. La localisation oropharyngée est très rare. Le diagnostic est orienté par l'imagerie, mais la confirmation reste toujours chirurgicale et anatomopathologique.

\section{REFERENCES}

1] Jchoo M., Kim Y-J, Jin H-R. A case of second branchial cleft cyst with oropharyngeal presentation J Korean Med sci 2002; 17:564-6.

3] William J. Développement de la tête, cou, des yeux et des oreilles. Embryologie humaine. Bruxelles 1996 : 311-39

4] Contencin Ph. Fistules et Kystes congénitaux du cou. Encycl Méd Chir.(ParisFrance)otorhinolaryngologie,1994;20-860-A-10,7p

5] Nicollas R, Guelfucci B, Roman S, Triglia JM. Congenital cysts and fistulas of the neck. Int J Pediatr Otorhinolaryngol 2000; 55:117-24.V10
6] Koch BL. Cystic malformations of the neck in children. Pediatr Radiol 2005; 35:463-77

7] Thaler FH,Tom JW,Handler SD. Second Branchial cleft anomalies presenting as pharyngeal masses. Otolaryngeal Head Neck Surg 1993; 109:941-4 